Correspondence

Ellie J. C. Goldstein

ejcgmd@aol.com
Received 20 August 2007

Accepted 29 November 2007

\section{Virulence characteristics of community-associated Staphylococcus aureus and in vitro activities of moxifloxacin alone and in combination against community-associated and healthcare-associated meticillin-resistant and -susceptible $S$. aureus}

\author{
Ellie J. C. Goldstein, ${ }^{1,2}$ Diane M. Citron, ${ }^{1}$ Yumi A. Warren, ${ }^{1}$ Kerin L. Tyrrell ${ }^{1}$ \\ and Michael J. Rybak ${ }^{3}$ \\ ${ }^{1}$ R. M. Alden Research Laboratory, Santa Monica, CA 90404, USA \\ ${ }^{2}$ UCLA School of Medicine, Los Angeles, CA 90095, USA \\ ${ }^{3}$ Anti-Infective Research Laboratory, College of Pharmacy and Health Sciences, Wayne State \\ University, Detroit, Ml 48201, USA
}

\begin{abstract}
The increasing prevalence of community-associated meticillin-resistant Staphylococcus aureus (CA-MRSA) poses a challenge for antimicrobial therapy of skin and soft tissue infections (SSTIs). To determine whether another antimicrobial agent might enhance the activity of moxifloxacin against CA-MRSA, this study analysed its activity alone and in chequerboard combination with doxycycline, rifampicin, clindamycin, trimethoprim, sulfamethoxazole/trimethoprim (SXT) and vancomycin against recent SSTI clinical isolates, and also characterized the isolates for Panton-Valentine leukocidin (PVL), agr groups, staphylococcal cassette chromosome mec (SSCmec) types and $\delta$-haemolysin production. For comparison, 25 strains of outpatient meticillin-susceptible S. aureus (MSSA), 24 strains of healthcare-associated (HA)-MRSA and six historical strains of vancomycin-intermediate $S$. aureus (VISA) were included. It was found that 21/25 CA-MRSA strains tested were PVL-positive, SSCmec type 4 and agr type 1, whilst 4/25 were PVL-negative, SSCmec type 2 and agr type 2. Two of the agr type 2 strains were negative for $\delta$-haemolysin but all other strains were positive. Moxifloxacin $\mathrm{MIC}_{50 / 90}$ values $(\mu \mathrm{g}$ $\mathrm{ml}^{-1}$ ) were $1 / 8$ for CA-MRSA, 4/32 for HA-MRSA and $\leqslant 0.03 / 1$ for MSSA and $\mathrm{MIC}_{50}$ of 2 for VISA. The D-test for inducible clindamycin resistance was positive for 3/27 CA-MRSA, 5/14 HA-MRSA and none of the MSSA isolates. In chequerboard studies, fractional inhibitory concentration indices (FICls) showed that most interactions were additive or indifferent $(\mathrm{FICl}$ value $>0.5$ to $\leqslant 2$ ) as follows: rifampicin $43 / 52$ strains, clindamycin $44 / 44$, SXT $44 / 47$, trimethoprim 41/42 and vancomycin 37/43. The $\mathrm{FICl}$ values for doxycycline were 3-6 for 32/34 strains, indicating antagonism, suggesting that it should not be used in combination with moxifloxacin.
\end{abstract}

\section{INTRODUCTION}

Community-associated meticillin-resistant Staphylococcus aureus (CA-MRSA) has become known as the 'most common identifiable cause' of skin and soft tissue infection (SSTI) across the USA (Moran et al., 2006) and has altered

Abbreviations: $\mathrm{CA}$, community-associated; $\mathrm{FICl}$, fractional inhibitory concentration index; HA, healthcare-associated; MRSA, meticillinresistant Staphylococcus aureus; MSSA, meticillin-susceptible Staphylococcus aureus; PVL, Panton-Valentine leukocidin; SCCmec, staphylococcal cassette chromosome mec; SSTI, skin and soft tissue infection; SXT, sulfamethoxazole/trimethoprim; VISA, vancomycin-intermediate Staphylococcus aureus. the clinician's antimicrobial selection. As there are no reliable, predictable patient characteristics for CA-MRSA infection, combination therapy is initiated empirically. Moxifloxacin is approved by the Food and Drug Administration and recommended by the Infectious Diseases Society of America (Stevens et al., 2005) for SSTIs; therefore, combined use of moxifloxacin with an anti-MRSA agent is a potential clinical consideration. In order to select the most appropriate agents, we evaluated the activity of six drugs combined with moxifloxacin against recent clinical isolates of CA-MRSA obtained from 33 patients with community-associated SSTIs. For comparison, we also studied 24 strains of healthcare-associated 
(HA)-MRSA, 25 strains of meticillin-susceptible S. aureus (MSSA) and six vancomycin-intermediate S. aureus (VISA) strains.

Jarraud et al. (2002) noted that CA-MRSA SSTIs are potentially more serious than SSTIs caused by other strains and that virulence factors such as Panton-Valentine leukocidin (PVL) may be a link between phylogenetics and virulence. In addition, Sakoulas et al. (2005) noted that there are 'sparse data' about associations of agr type II CAMRSA with 'infections with particular infectious syndromes'. Consequently, we selected the first 25 of our 33 CA-MRSA isolates, and determined the virulence genes and expression of PVL, SSCmec type, agr type and $\delta$ haemolysis production.

\section{METHODS}

Test strains included 33 consecutive isolates of MRSA and 25 isolates of MSSA recovered from SSTIs in outpatients between 30 September and 17 October 2005; 24 strains of HA-MRSA from blood, respiratory samples and wounds collected between 20 October and 5 December 2005; and 6 historical strains of VISA obtained from Professor K. Hiramatsu (Juntendo University, Tokyo, Japan).

Determination of MICs and chequerboard synergy studies were carried out in cation-adjusted Mueller-Hinton broth with moxifloxacin alone and in combination with doxycycline, rifampicin, clindamycin, trimethoprim, sulfamethoxazole/trimethoprim (SXT) and vancomycin. MICs without synergy studies were determined for levofloxacin and erythromycin (CLSI, 2006). Moxifloxacin concentrations were $0.03-4 \mu \mathrm{g} \mathrm{ml}^{-1}$ plus each of the other agents at four or five clinically appropriate concentrations. The fractional inhibitory concentration index (FICI) was calculated for all combinations (Krogstad \& Moellering, 1986). Erythromycin-resistant, clindamycinsusceptible strains were tested for inducible resistance by the D-test. For quality control, we used S. aureus ATCC 29213 and ATCC 43300

Staphylococcal cassette chromosome mec (SCCmec) type was determined by multiplex PCR methods as described previously by Zhang et al. (2005). The PVL genes, lukS-PV and lukF-PV, were detected by PCR using primers described by Lina et al. (1999). Identification of agr group was accomplished using specific primers. Clinical S. aureus isolates were studied using PCR and agr groupspecific primers as follows: agr group I, 5'-CACTTATCATCAAAGAGCC-3' and 5'-CCACTAATTATAGCTGG-3; agr group II, 5' GTAGAGCCGTATTGATTCC-3' and $5^{\prime}$-TATTTCATCTCTTTAAGG-3'; agr group III, $\operatorname{agrC}$ of class III: $5^{\prime}$-CTGCATTTATTAGTGGAATACG-3' and 5'-GTTTCATTTCTTTAAGAG-3' ${ }^{\prime}$; agr group IV, 5'-GCCGCGAGCTTGGGAG-3' and 5'-GAAGATACGTGGCAAACTGGT-3'. After amplification for 30 cycles $(30 \mathrm{~s}$ of denaturation at $94{ }^{\circ} \mathrm{C}, 30 \mathrm{~s}$ of annealing at $55^{\circ} \mathrm{C}$ and $1 \mathrm{~min}$ of extension at $72{ }^{\circ} \mathrm{C}$ ), the PCR products were resolved by electrophoresis through $1.5 \%$ agarose gels followed by ethidium bromide staining and analysis. $S$. aureus ATCC 49775 was used as a positive control strain for all PCR runs. Expression of the agr gene cluster was determined by $\delta$ haemolysin production using a strain of $S$. aureus (RN4220) that produces a large zone of $\beta$-haemolysis without interference by $\alpha$ - or $\delta$ haemolysins. Briefly, RN4220 was streaked vertically and test strains were streaked perpendicular to it on sheep blood agar plates. S. aureus strains RN6607 and RN9120 were used as positive and negative controls, respectively. Evidence for enhanced haemolysin production was examined at the intersection of growth of the two organisms (Sakoulas et al., 2003).

\section{RESULTS}

The determined MIC and FICI values are summarized in Table 1 . The moxifloxacin $\mathrm{MIC}_{50 / 90}$ values $\left(\mu \mathrm{g} \mathrm{ml}^{-1}\right)$ were $1 / 8$ for CA-MRSA, $4 / 32$ for HA-MRSA and $\leqslant 0.03 / 1$ for MSSA, and $\mathrm{MIC}_{50}$ of 2 for VISA. The D-test was positive for $3 / 27(11 \%)$ CA-MRSA strains that were resistant to erythromycin but appeared to be susceptible to clindamycin, 5/14 (36\%) of the HA-MRSA strains and none of the MSSA strains.

In chequerboard studies, FICI values were calculated and most interactions were additive or indifferent (FICI $>0.5$ to $\leqslant 2$ ); however, the FICI values for doxycycline were 3-6 for $32 / 34$ strains, indicating antagonism. Approximately $10 \%$ of moxifloxacin-resistant strains (MIC $>0.5 \mu \mathrm{g} \mathrm{ml}^{-1}$ ) exhibited a reduction in their MIC to $\leqslant 0.5 \mu \mathrm{g} \mathrm{ml}^{-1}$ with the addition of clindamycin and SXT and $\sim 25 \%$ with the addition of vancomycin, rifampicin and trimethoprim alone. There was no reduction with the addition of doxycycline.

Of the 25 CA-MRSA strains tested, 21 (84\%) were PVLpositive, SSCmec type 4 and agr type 1, suggesting a dominant geographical clone. Four out of 25 (16\%) were PVL-negative, SSCmec type 2 and agr type 2. Two of these four were negative for $\delta$-haemolysin production, but all other strains were positive.

\section{DISCUSSION}

The association of virulence factors and clinical conditions has been studied infrequently (Sakoulas et al., 2005). Jarraud et al. (2002) examined 198 strains, but only four of these were from patients with cellulitis and/or myositis, with one from agr group I and three from agr group III. Vandenesch et al. (2003) studied 117 CA-MRSA isolates from various countries worldwide by PFGE and multilocus sequence typing and noted 'distinct genetic backgrounds associated with each geographic origin' that were not related to the genetic background of the HA-MRSA strains. They found that SSCmec IV and PVL loci were common to all locations and that, although $97 \%$ were agr type 3, those from the USA were generally agr type 1 (USA 300), which is in accord with our findings. Diep et al. (2006) studied the evolution of CA-MRSA isolates from San Francisco over 8.5 years and found that the current epidemic phenotype strain was associated with the horizontal acquisition of SSCmec IV, PVL genes and also enterotoxin $\mathrm{Q}$ and K genes. Recently, S. aureus was isolated from $76 \%$ of patients $(320 / 422)$ with SSTIs who presented to 11 university-affiliated emergency departments. MRSA accounted for $59 \%$ (range 15-74\%) of these isolates with USA 300 accounting for $97 \%$ and SSCmec type IV and PVL being present in $98 \%$ of CA-MRSA isolates (Moran et al., 2006). This was slightly higher than in our results, suggesting some diversity in SSTIs.

Our in vitro data showed that moxifloxacin was two to four times more active than levofloxacin on a weight basis, 
Table 1. In vitro activity of moxifloxacin and other antimicrobial agents alone and in combination, and their $\mathrm{FICl}$ values against $\mathrm{MRSA}$, MSSA and VISA strains

\begin{tabular}{|c|c|c|c|c|c|c|c|c|}
\hline \multirow{2}{*}{$\begin{array}{l}\text { Antibiotic (susceptible } \\
\text { breakpoint, } \mu \mathrm{g} \mathrm{ml}^{-1} \text { ) }\end{array}$} & \multicolumn{3}{|c|}{$\operatorname{MIC}\left(\mu \mathrm{g} \mathrm{ml}^{-1}\right)$} & \multirow{2}{*}{$\begin{array}{l}\text { Susceptible } \\
\quad(\%)\end{array}$} & \multirow{2}{*}{$\begin{array}{c}\text { D-test } \\
\text { positive }^{\star}\end{array}$} & \multicolumn{2}{|c|}{ FICI } & \multirow{2}{*}{$\begin{array}{c}\text { Moxifloxacin-resistant strains } \\
\text { with moxifloxacin MIC } \leqslant 0.5 \\
\text { in chequerboard }(\%)\end{array}$} \\
\hline & Range & $\mathrm{MIC}_{50}$ & $\mathrm{MIC}_{90}$ & & & Range (no.) & Median & \\
\hline CA-MRSA (33 isolates) & & & & & $3 / 27$ & & & \\
\hline Moxifloxacin $(\leqslant 0.5)$ & $\leqslant 0.03-32$ & 1 & 8 & 15.2 & & & & \\
\hline Doxycycline $(\leqslant 4)$ & $0.125-4$ & 0.125 & 0.25 & 100 & & $2-5(20)$ & 3 & 0 \\
\hline Trimethoprim $(\leqslant 8)$ & $0.5-4$ & 2 & 2 & 100 & & $0.5-3(23)$ & 1 & 28.6 \\
\hline $\operatorname{SXT}(\leqslant 2 / 38)$ & $0.06-0.25$ & 0.06 & 0.125 & 100 & & $0.5-3(26)$ & 1 & 10.3 \\
\hline Clindamycin $(\leqslant 0.5)$ & $0.125->128$ & 0.125 & $>128$ & 93.9 & & $0.5-2(25)$ & 2 & 10.7 \\
\hline Erythromycin $(\leqslant 0.5)$ & $0.5->128$ & 128 & $>128$ & 9.1 & & NT & & \\
\hline Rifampicin $(\leqslant 1)$ & $0.008-0.015$ & 0.008 & 0.008 & 100 & & $0.75-3(23)$ & 2 & 25 \\
\hline Vancomycin $(\leqslant 2)$ & $0.5-1$ & 1 & 1 & 100 & & $0.56-3(23)$ & 2 & 23.8 \\
\hline Levofloxacin $(\leqslant 1)$ & $\leqslant 0.5->16$ & 4 & $>16$ & 15.1 & & NT & & \\
\hline HA-MRSA ( 24 isolates) & & & & & $5 / 14$ & & & \\
\hline Moxifloxacin $(\leqslant 0.5)$ & $1-32$ & 4 & 32 & 0 & & & & \\
\hline Doxycycline $(\leqslant 4)$ & $0.06-2$ & 0.125 & 0.25 & 100 & & $3-6(11)$ & 5 & 0 \\
\hline Trimethoprim $(\leqslant 8)$ & $0.5-4$ & 2 & 4 & 100 & & $0.31-3(16)$ & 2 & 19 \\
\hline SXT $(\leqslant 2 / 38)$ & $0.06-0.25$ & 0.125 & 0.125 & 100 & & $0.27-2(18)$ & 1 & 22.2 \\
\hline Clindamycin $(\leqslant 0.5)$ & $\leqslant 0.06->128$ & 0.125 & $>128$ & 62.5 & & $0.13-2(14)$ & 0.51 & 50 \\
\hline Erythromycin $(\leqslant 0.5)$ & $0.5->128$ & $>128$ & $>128$ & 0 & & NT & & \\
\hline Rifampicin $(\leqslant 1)$ & $0.008-0.03$ & 0.008 & 0.015 & 100 & & $1-3(18)$ & 2 & 4.2 \\
\hline Vancomycin $(\leqslant 2)$ & $0.5-1$ & 1 & 1 & 100 & & $0.75-3(16)$ & 2 & 4.2 \\
\hline Levofloxacin $(\leqslant 1)$ & $4->16$ & $>16$ & $>16$ & 0 & & NT & & \\
\hline MSSA (25 isolates) & & & & & $0 / 4$ & & & \\
\hline Moxifloxacin $(\leqslant 0.5)$ & $\leqslant 0.03-4$ & $\leqslant 0.03$ & 1 & 88 & & & & \\
\hline Doxycycline $(\leqslant 4)$ & $0.125-2$ & 0.25 & 0.25 & 100 & & $3-5(3)$ & 3 & 0 \\
\hline Trimethoprim $(\leqslant 8)$ & $2-4$ & 2 & & 100 & & $1-2(3)$ & 1 & 33.3 \\
\hline SXT $(\leqslant 2 / 38)$ & $0.125->8$ & 0.125 & 0.25 & 92 & & $0.38-2(3)$ & 1.5 & 0 \\
\hline Clindamycin $(\leqslant 0.5)$ & $\leqslant 0.06->128$ & 0.125 & 0.25 & 96 & & $1-2(5)$ & 1 & 0 \\
\hline Erythromycin $(\leqslant 0.5)$ & $0.5->128$ & 0.5 & $>128$ & 76 & & NT & & \\
\hline Rifampicin $(\leqslant 1)$ & $0.008-0.015$ & 0.008 & 0.015 & 100 & & $1-2(12)$ & 1.5 & 0 \\
\hline Vancomycin $(\leqslant 2)$ & $\leqslant 0.03-1$ & 1 & 1 & 100 & & $2-2$ & 2 & 0 \\
\hline Levofloxacin $(\leqslant 1)$ & $\leqslant 0.5->16$ & $\leqslant 0.5$ & 4 & 88 & & NT & & \\
\hline VISA (6 isolates) & & & & & - & & & \\
\hline Moxifloxacin $(\leqslant 0.5)$ & $1-4$ & 2 & - & 0 & & & & \\
\hline Doxycycline $(\leqslant 4)$ & $4-8$ & 4 & - & 83 & & - & - & \\
\hline Trimethoprim $(\leqslant 8)$ & $2->16$ & 4 & - & 50 & & $2-2(4)$ & 2 & \\
\hline $\operatorname{SXT}(\leqslant 2 / 38)$ & $0.125->8$ & 0.125 & - & 50 & & $2-2(4)$ & 2 & \\
\hline Clindamycin $(\leqslant 0.5)$ & $>128->128$ & $>128$ & - & 0 & & NT & & \\
\hline Erythromycin $(\leqslant 0.5)$ & $>128->128$ & $>128$ & - & 0 & & NT & & \\
\hline Rifampicin $(\leqslant 1)$ & $0.008->8$ & 2 & - & 33.3 & & $2-3(6)$ & - & \\
\hline Vancomycin $(\leqslant 2)$ & $1-8$ & 4 & - & 33.3 & & NT & & \\
\hline Levofloxacin $(\leqslant 1)$ & $4-16$ & 8 & - & 0 & & NT & & \\
\hline
\end{tabular}

NT, Not tested.

${ }^{*}$ D-test for inducible clindamycin resistance.

which might be the result of a single mutation in $g r l A$ or gyrA, as postulated by Noguchi et al. (2005). As the time above the MIC is a key parameter in the killing of S. aureus, the protein binding of moxifloxacin versus levofloxacin (30$50 \%$ vs $24-38 \%$ ) and the time above the MIC (moxifloxacin has a half-life of 10-14 h vs $7 \mathrm{~h}$ for levofloxacin) would give advantage to moxifloxacin (although one should only consider free drug concentrations). Clinically, several studies have suggested the utility of a fluoroquinolone plus rifampicin for the therapy of MSSA infections. Zimmerli et al. (1998) treated 33 patients with ciprofloxacin plus rifampicin for orthopaedic prosthesis infections and had a $100 \%$ cure rate in the experimental group compared with $58 \%$ in the control group (ciprofloxacin plus placebo) 
$(P<0.02)$. Schrenzel et al. (2004) treated 104 patients with deep-seated $S$. aureus infections, mostly MSSA, and found that a fluoroquinolone plus rifampicin was effective in $78 \%$ of cases. Our data support these studies and suggest that moxifloxacin plus rifampicin would be effective for MSSA infections.

In our study, $15.2 \%$ of CA-MRSA isolates were susceptible to $\leqslant 0.5 \mu \mathrm{g}$ moxifloxacin $\mathrm{ml}^{-1}$ and $63.6 \%$ to $1.0 \mu \mathrm{g}$ moxifloxacin $\mathrm{ml}^{-1}$. It is important to note that there is disparity between Food and Drug Administration and CLSI suggested breakpoints, but, despite in vitro activity, clinically moxifloxacin is not indicated for use in any MRSA infection, and prudent practice would suggest the co-administration of a second agent if MRSA were suspected. In our study, up to $29 \%$ of moxifloxacinresistant isolates decreased their moxifloxacin MICs to $\leqslant 0.5 \mu \mathrm{g} \mathrm{ml}{ }^{-1}$ with the addition of a second agent, especially trimethoprim alone.

We found that $100 \%$ of our CA-MRSA isolates were susceptible to doxycycline, SXT, trimethoprim, vancomycin and rifampicin, and $93.9 \%$ to clindamycin, but only $9.1 \%$ to erythromycin. This is similar to the results of Moran et al. (2006), who reported that $95 \%$ of their MRSA isolates were susceptible to clindamycin, $6 \%$ to erythromycin, $60 \%$ to fluoroquinolones, $100 \%$ to SXT and $92 \%$ to tetracyclines. In contrast, Buck et al. (2005) noted that 201/517 SSTI CA-MRSA isolates (39\%) from patients in Minnesota were susceptible to erythromycin and 433/517 $(84 \%)$ to ciprofloxacin, whilst susceptibility to other agents was more typical (clindamycin $92 \%$, rifampicin $99 \%$, tetracycline $91 \%$ and SXT 99\%). However, they found that, unlike CA-MRSA isolates, only $77 \%$ (44/57) of invasive HA-MRSA isolates were susceptible to clindamycin.

Differences between the susceptibilities of MRSA and MSSA to moxifloxacin were noted by Metzler et al. (2004), who found that moxifloxacin was more potent $\left(\mathrm{MIC}_{90}\right.$ $0.06 \mathrm{mg} \mathrm{l}^{-1}$ ) than levofloxacin ( $\mathrm{MIC}_{90} 0.25 \mathrm{mg} \mathrm{l}^{-1}$ ) against MSSA isolates, and this was also true at mutation-prevention concentrations (MPCs) (moxifloxacin $0.25 \mathrm{mg} \mathrm{l}^{-1}$ vs levofloxacin $1 \mathrm{mg} \mathrm{l}^{-1}$ ); however, $\mathrm{MIC}_{90}$ and $\mathrm{MPC}_{90}$ were higher for both drugs against the MRSA isolates. Metzler et al. (2004) did not look at the comparative resistance prevention of moxifloxacin versus levofloxacin in combination with SXT or rifampicin. In our study, moxifloxacin $\mathrm{MIC}_{50 / 90}$ values were lowest for MSSA, intermediate for CA-MRSA and highest for HA-MRSA; except for levofloxacin, the activity of the other agents against HA-MRSA was similar to that for CA-MRSA. In contrast, moxifloxacin was active against 23/25 (92\%) of our MSSA isolates at $<1 \mu \mathrm{g} \mathrm{ml}{ }^{-1}$ (range $\leqslant 0.03-4$ ); clindamycin and erythromycin were active against 96 and $76 \%$, respectively.

For the six VISA strains that we tested, the moxifloxacin MICs were $1-4 \mu \mathrm{g} \mathrm{ml}^{-1}$. SXT was active against $50 \%$, doxycycline against $83 \%$ and rifampicin against $33.3 \%$ of strains. All were clindamycin- and erythromycin-resistant. This suggests that the VISA strains are multidrug-resistant and that selection of antimicrobial therapy must be based on individual susceptibility testing.

Our study shows that most interactions with moxifloxacin against MRSA were either additive or indifferent (FICI $>0.5$ to $\leqslant 2$ ) for rifampicin, clindamycin, SXT, trimethoprim and vancomycin. Of note is the extremely high activity of rifampicin alone against all MRSA and MSSA isolates; future studies to determine whether or not moxifloxacin prevents the emergence of rifampicin-resistant $S$. aureus would be of interest. The FICI values for doxycycline were 3-6 for 32/34 strains, indicating antagonism and suggesting that this combination should be avoided.

\section{ACKNOWLEDGEMENTS}

This study was sponsored in part by a grant from Schering-Plough Corporation. We also thank Alice E. Goldstein, Judee Knight and H. Fernandez for assistance.

\section{REFERENCES}

Buck, J. M., Como-Sabetti, K., Harriman, K. H., Danila, R. N., Boxrud, D. J., Glennen, A. \& Lynfield, R. (2005). Community-associated methicillin-resistant Staphylococcus aureus, Minnesota, 2000-2003. Emerg Infect Dis 11, 1532-1538.

CLSI (2006). Methods for Dilution Antimicrobial Susceptibility Tests for Bacteria that Grow Aerobically; approved standard, 7th edn. Document M7-A7. Wayne, PA: Clinical and Laboratory Standards Institute.

Diep, B. A., Carleton, H. A., Chang, R. F., Sensabaugh, G. F. \& Perdeau-Remmington, F. (2006). Roles of 34 virulence genes in the evolution of hospital- and community-associated strains of methicillin-resistant Staphylococcus aureus. J Infect Dis 193, 1495-1503.

Jarraud, S., Mougel, C., Thiolouse, J., Lina, G., Meugnier, H., Forey, F., Nesme, X., Etienne, J. \& Vandenesch, F. (2002). Relationships between Staphylococcus aureus genetic background, virulence factors, agr groups (alleles) and human disease. Infect Immun 70, 631-641.

Krogstad, D. J. \& Moellering, R. C., Jr (1986). Antimicrobial combinations. In Antibiotics in Laboratory Medicine, 2nd edn. Edited by V. Lorian. Baltimore, MD: Williams and Wilkins.

Lina, G., Piémont, Y., Godail-Gamot, F., Bes, M., Peter, M. O., Gauduchon, V., Vandenesch, F. \& Etienne, J. (1999). Involvement of Panton-Valentine leukocidin-producing Staphylococcus aureus in primary skin infections and pneumonia. Clin Infect Dis 29, 1128-1132.

Metzler, K., Hansen, G. M., Hedlin, P., Harding, E., Drlica, K. \& Blondeau, J. M. (2004). Comparison of minimal inhibitory and mutant prevention drug concentrations of 4 fluoroquinolones against clinical isolates of methicillin-susceptible and -resistant Staphylococcus aureus. Int J Antimicrob Agents 24, 161-167.

Moran, G. J., Krishnadasan, A., Horowitz, R. J., Fosheim, G. E., McDougal, L. K., Carey, R. B. \& Talan, D. A. (2006). Methicillinresistant $S$. aureus infections among patients in the emergency department. N Engl J Med 355, 666-674.

Noguchi, N., Okihara, T., Namaki, Y., Kumaki, Y., Yamanake, Y., Koyama, M., Wakasugi, K. \& Sasatsu, M. (2005). Susceptibility and resistance genes to fluoroquinolones in methicillin-resistant 
Staphylococcus aureus isolated in 2002. Int J Antimicrob Agents 25, 374-379.

Sakoulas, G., Eliopoulos, G. M., Moellering, R. C., Jr, Novick, R. P., Venkataraman, L., Wennerstein, C., DeGirolami, P. C., Schwaber, M. J. \& Gold, H. S. (2003). Staphylococcus aureus accessory gene regulator $(a g r)$ group II: is there a relationship to the development of intermediate-level glycopeptide resistance? J Infect Dis 187, 929-938.

Sakoulas, G., Eliopoulos, G. M., Fowler, V. G., Moellering, R. C., Jr, Novick, R. P., Lucindo, N., Yeaman, M. R. \& Bayer, A. S. (2005). Reduced susceptibility of Staphylococcus aureus to vancomycin and platelet microbicidal protein correlates with defective autolysis and loss of accessory gene regulator (agr) function. Antimicrob Agents Chemother 49, 2687-2692.

Schrenzel, J., Harbarth, S., Schockmel, G., Genné, D., Bregenzer, T., Flueckiger, U., Petignat, C., Jacobs, F., Francioli, P. \& other authors (2004). A randomized clinical trial to compare fleroxacin-rifampicin with flucloxacin or vancomycin for the treatment of staphylococcal infections. Clin Infect Dis 39, 1285-1292.
Stevens, D. L., Bisno, A. L., Chambers, H. F., Everett, E. D., Dellinger, P., Goldstein, E. J. C., Gorbach, S. L., Hirschmann, J. V., Kaplan, E. L. \& other authors (2005). Skin and soft tissue infections: diagnosis and management guidelines. Clin Infect Dis 41, 1373-1406.

Vandenesch, F., Nami, T., Enright, M. C., Lina, G., Nimmo, G. R., Heffernan, H., Liassine, N., Bes, M., Greenland, T. \& other authors (2003). Community-acquired methicillin-resistant Staphylococcus aureus carrying Panton-Valentine leucocidin genes: worldwide emergence. Emerg Infect Dis 9, 978-984.

Zhang, K., McClure, J. A., Elsayed, S., Louie, T. \& Conly, J. M. (2005). Novel multiplex PCR assay for characterization and concomitant subtyping of staphylococcal cassette chromosome SSCmec types I to V in methicillin-resistant Staphylococcus aureus. J Clin Microbiol 43, 5026-5033.

Zimmerli, W., Widmer, A. F., Blatter, M., Frei, R. \& Ochsner, P. E. (1998). Role of rifampicin for treatment of orthopedic implantrelated staphylococcal infections: a randomized controlled trial. JAMA 279, 1537-1541. 